\title{
The naked truth: Sphynx and Devon Rex cat breed mutations in KRT71
}

\author{
Barbara Gandolfi · Catherine A. Outerbridge • \\ Leslie G. Beresford · Jeffrey A. Myers • Monica Pimentel • \\ Hasan Alhaddad • Jennifer C. Grahn • Robert A. Grahn • \\ Leslie A. Lyons
}

Received: 7 July 2010/ Accepted: 13 September 2010/Published online: 16 October 2010

(C) The Author(s) 2010. This article is published with open access at Springerlink.com

\begin{abstract}
Hair is a unique structure, characteristic of mammals, controlling body homeostasis, as well as cell and tissue integration. Previous studies in dog, mouse, and rat have identified polymorphisms in Keratin 71 (KRT71) as responsible for the curly/wavy phenotypes. The coding sequence and the $3^{\prime}$ UTR of KRT71 were directly sequenced in randomly bred and pedigreed domestic cats with different pelage mutations, including hairless varieties. A SNP altering a splice site was identified in the Sphynx breed and suggested to be the hairless $(h r)$ allele, and a complex sequence alteration, also causing a splice variation, was identified in the Devon Rex breed and suggested to be the curly ( $r e$ allele. The polymorphisms were genotyped in approximately 200 cats. All the Devon Rex were homozygous for the complex alterations and most of the Sphynx were either homozygous for the $h r$ allele or compound heterozygotes with the Devon-associated re allele, suggesting that the phenotypes are a result of the identified SNPs. Two Sphynx carrying the proposed $h r$ mutation did not carry the Devon-associated alteration. No other causative mutations for eight different rexoid and
\end{abstract}

Electronic supplementary material The online version of this article (doi:10.1007/s00335-010-9290-6) contains supplementary material, which is available to authorized users.

B. Gandolfi - L. G. Beresford - J. A. Myers - M. Pimentel · H. Alhaddad · J. C. Grahn · R. A. Grahn · L. A. Lyons ( $\square)$ Department of Population Health and Reproduction,

School of Veterinary Medicine, University of California-Davis,

1114 Tupper Hall, Davis, CA 95616, USA

e-mail: lalyons@ucdavis.edu

\section{A. Outerbridge}

Department of Veterinary Medicine and Epidemiology,

School of Veterinary Medicine, University of California-Davis,

Davis, CA 95616, USA hairless cat phenotypes were identified. The allelic series $K R T 71^{+}>K R T 71^{h r}>K R T 71^{r e}$ is suggested.

\section{Introduction}

A mammal's pelage is generally one of its first noticeable attributes and is aesthetically pleasing. Coat color and pelage types are often selected as specific traits to develop new domesticated mammalian breeds. Frequently, the causative mutations for these phenotypes are inherited as single-gene, autosomal traits, but genetic modifiers often determine the preferred "refined" phenotype to meet breed standards. Although the genetic basis of several coat colors has been characterized in cats (Hatzfeld and Weber 1990; Ishida et al. 2006; Lyons et al. 2005a, b; Schmidt-Kuntzel et al. 2005), and the major gene controlling fur length, FGF5, has been determined (Cadieu et al. 2009; Drogemuller et al. 2007; Housley and Venta 2006; Kehler et al. 2007), relatively little is known about the genes influencing variation in coat growth and curl, i.e., rexoid coats. Curly pelage is an easily recognized trans-species coat anomaly, and several detailed studies in various mammalian species, including mice (Dunn 1937; Runkel et al. 2006; Taylor et al. 2000), rats (Kuramoto et al. 2010; Robinson 1981), and dogs (Cadieu et al. 2009) have identified causative genes and mutations.

At least nine rex-type pelage mutations are documented in the domestic cat (Supplementary Table 1, Fig. 1). A few other rexoid mutations are mentioned in cat lay literature but are not scientifically documented, and breeds were not successfully established with the phenotypes (Morris 1999). Three rexoid mutations are the hallmark of traditional and well-established breeds, including the American Wirehair, Cornish Rex (Searle and Jude 1956), and the 
Fig. 1 Hairless and curly phenotypes on the domestic cat. a Hairless phenotype in a Sphynx cat, characterized by lack of hair with a well-formed bulb. b Curly phenotype in a Devon Rex cat characterized by a short and soft coat, generally missing the guard hairs
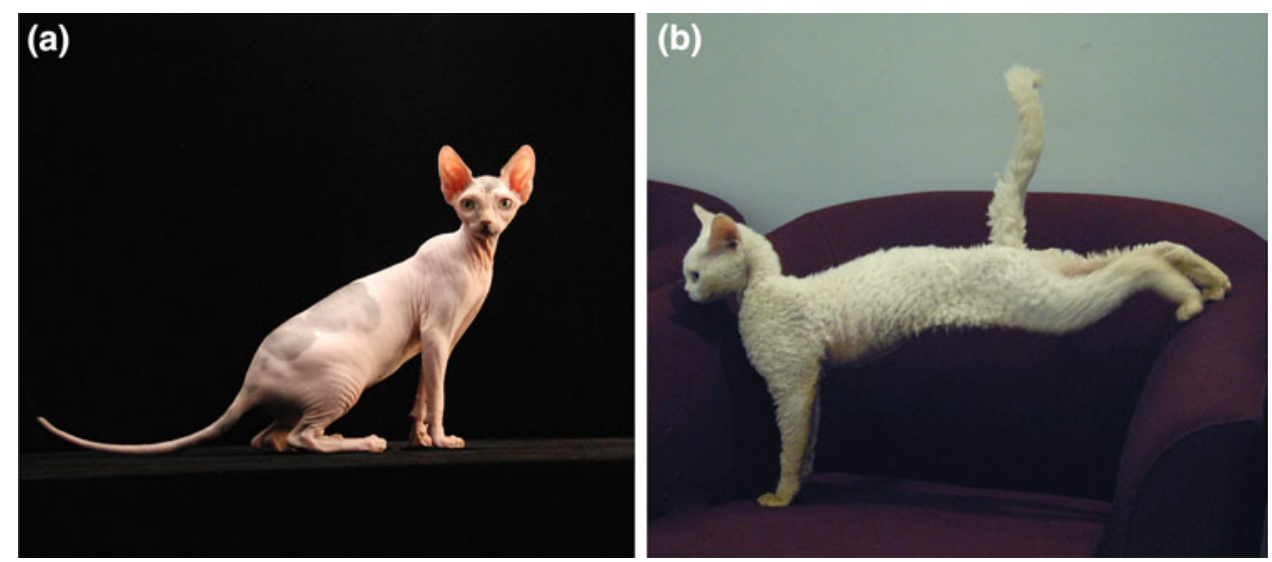

Devon Rex (Robinson 1969). Several other curly-coated cats are not scientifically documented but are gaining popularity and in development as separate breeds, including two autosomal dominant rexoids La Perm and Selkirk Rex and one autosomal recessive rexoid, the Tennessee Rex.

The two well-established rexoid breeds, the Devon Rex, $r e$, and the Cornish Rex, $r$, are scientifically documented as different autosomal recessive loci (Robinson 1969), the Devon Rex having more variable expression than the Cornish Rex. For both breed phenotypes, the coat of a rexoid mutant is short, without guard hairs, and with a reduction in length and thickness of the remaining fibers (down and awn hair). The Devon Rex hair is abnormal and the majority is broken distally at their thinnest diameter, while the intact hairs are bluntly pointed instead of showing the fine tapering point of the normal hair fiber (Robinson 1969). Curly coats are a defining feature of the breeds, thus, most cats are fixed for the mutations.

Devon Rex has been used in the development of the hairless (hr) Sphynx breed and the hairless mutation is considered to be allelic to the Devon rexoid mutation (Robinson 1973). The Sphynx hairless is also considered an autosomal recessive trait and is one of the more distinctive novelty breeds, steadily increasing in popularity. Only two hair types seem to be present: guard hairs of irregular diameter and curved and thinner hairs. Thus, Sphynx is not completely hairless, having a fine down on the body and some light and wavy hair often present on the nose, tail, and toes. All the hairs lack a well-formed bulb, which probably explains why hairs are so easily dislodged (Robinson 1973), causing a lack of hair on the body but not a complete lack of hair production.

Recently, genome-wide SNP association studies led to candidate gene screening for the curly/wavy coat of the Portuguese Water Dog. A SNP in keratin-71 (KRT71), which encodes a protein that is predominantly expressed in the inner root sheath (IRS) of the hair follicle (Langbein et al. 2002), was shown to cause a nonsynonymous mutation, c. $451 \mathrm{~A}>\mathrm{T}$, in exon 2, producing a p.Arg151Trp amino acid alteration (Cadieu et al. 2009). Beside dogs, mutations in KRT71 have been described in curly-coated mice (Dunn 1937; Runkel et al. 2006; Taylor et al. 2000) and rats (Kuramoto et al. 2010), and an autosomal recessive form has been described in Hereford cattle (A. D. Markey, unpublished).

For the domestic cat, genomic sequence for KRT71 was publicly available except for exon 9 and the $3^{\prime}$ UTR. A variety of curly-coated, wire-haired, and hairless cat breeds $(N=10)$ were scanned for mutations in the coding sequence of KRT71. Mutations concordant with the curly (rexoid) coat of the Devon Rex and the hairless coat of the Sphynx were identified and appear to be causative mutations for the pelage phenotypes. They are unique mutations within these breeds. The characterization of these mutants demonstrates the importance of KRT71 in the formation of linear and normal IRS intermediate filaments. Mutations in the coding region of KRT71 were not identified in the two additional hairless breeds and the six other wired and rexoid cat breeds.

\section{Materials and methods}

\section{Sample collection}

Phenotype descriptions of the cats were determined from personal interviews of the breeders and by direct observation. DNA samples were collected from the cats noninvasively using buccal swabs at cat shows and the owners' homes. DNA was isolated from the buccal swab using the QIAamp DNA mini kit (Qiagen, Valencia, CA).

\section{KRT71 genomic analysis}

All nine exons of KRT71 were amplified by PCR from genomic DNA of 24 cats (Supplementary Table 2), including seven rexoid breeds, three hairless breeds, and a control, domestic shorthair. Publicly available sequences 
from various species were aligned for the gene KRT71, including Homo sapiens (NM_033448), Canis familiaris (XM_854730.1), and Mus musculus (NM_019956) and the partial sequence for the domestic cat, Felis catus (ENSFCAT00000000287), to identify the exons. F. catus KRT71 can be found on GeneScaffold_3321: 736,457-745.383. Primers (Operon, Huntsville, AL) were designed using Primer3plus (http://www.bioinformatics.nl/cgi-bin/primer 3 plus/primer3plus.cgi) in the intronic regions when possible, flanking each identified exon. Primers were tested for efficient product amplification on a DNA Engine Gradient Cycler (MJ Research, GMI, Ramsey, MN) and the final magnesium, temperature conditions, and amplicon size for each primer pair are shown in Supplementary Table 3. PCR and thermocycling conditions were conducted as previously described (Bighignoli et al. 2007). The PCR products with appropriate lengths were purified using the ExoSap (USB, Cleveland, $\mathrm{OH}$ ) enzyme per the manufacturer's recommendations. Purified genomic products were directly sequenced in both directions using BigDye Terminator Sequencing Kit v3.1 (Applied Biosystems, Carlsbad, CA), purified with Illustra Sephadex G-50 (GE Healthcare, Piscataway, NJ) according to the manufacturer's recommendations, and electrophoretically separated on an ABI 3730 DNA analyzer (Applied Biosystems). Sequences were verified and aligned using the software Sequencher version 4.8 (Gene Codes Corp., Ann Arbor, MI) and edited with BioEdit version 7.0.9.0 (Hall 1999)

\section{KRT71 mRNA analysis}

Skin biopsies from a control cat, a Sphynx, and a Devon Rex were provided by the UC Davis School of Veterinary Medicine, Dermatological Service. The tissues were stored in RNAlater (Qiagen), and RNA was isolated using PureLink RNA mini Kit (Invitrogen, Carlsbad, CA). Complementary DNA templates were synthesized by reverse transcription of $1 \mu \mathrm{g}$ of mRNA from hair bulbs of the control cat with the polydT primer to obtain exons 8 and 9 and the $3^{\prime}$ UTR of KRT71, from $1 \mu \mathrm{g}$ of skin mRNA using the KRT71-5RcDNA primer to obtain exons $1-5$ in the Sphynx, and from $1 \mu \mathrm{g}$ of skin mRNA using the KRT71-8RcDNA primer to obtain exon 1-8 in the Devon Rex with SuperScript III (Invitrogen) (Supplementary Table 3). PCR products were generated according to the manufacturer's recommendations using the primers GeneRacer3R (Invitrogen) and KRT71-8FcDNA for the hair sample and KRT71-ex5RcDNA, KRT71ex8RcDNA, and KRT71-ex1FcDNA for the skin samples (Supplementary Table 3). Thermocycle conditions were conducted as previously described (Bighignoli et al. 2007). Genomic primers were designed in the $3^{\prime}$ UTR region to sequence the cats used for the genomic analysis of KRT71 (Supplementary Table 3).

\section{KRT71 SNP genotyping}

An RFLP assay was performed to screen a possible causative KRT71 intron 4 polymorphism in 94 cats represented by randomly bred cats and all available wire, rexoid, and hairless breeds (Supplementary Table 1). The G/A substitution at the first base of intron 4 alters a normally present BceAI (ACGGC) restriction enzyme recognition site. Approximately $20 \mu \mathrm{l}$ of amplified product was digested with $2 \mathrm{U}$ of BceAI (New England Biolabs Inc., Beverly, MA) at $37^{\circ} \mathrm{C}$ overnight followed by inactivation of the enzyme at $65^{\circ} \mathrm{C}$ for $10 \mathrm{~min}$. The primers generated an amplification product of $832 \mathrm{bp}$ that produced 436- and 396-bp restriction fragment products using BceAI when the G/A SNP was absent. The complete digestion reaction was analyzed on $2 \%$ agarose gels as described above.

A second potentially causative polymorphism was an 81-bp deletion followed by two insertions of 8 and 1 bp starting at the $5^{\prime}$ end of intron 6 and involving exon 7 . A PCR reaction using the KTR71-ex7F2 and KRT71ex7R2 primers (Supplementary Table 3) was used to verify the genotype in 189 cats, including randomly bred cats and the wire, rexoid, and hairless breeds (Table 1). The predicted size of the wild-type allele was 557 bp and $485 \mathrm{bp}$ for the mutant. The genotype was predicted visually by the different length of the amplicon after a run on a $2 \%$ agarose gel.

\section{Results}

\section{KRT71 sequence analysis}

The entire KTR71 coding sequence (CDS) and the $3^{\prime}$ UTR were analyzed for 24 cats (Supplementary Table 2) representing all the rexoid and hairless variants (Fig. 1). A prediction of the feline KRT71 exon sizes was obtained using the human genomic sequence. In human and dog, KRT71 has only one known isoform and the CDS length is 1572 and $1578 \mathrm{bp}$, respectively, assuming the putative start of methionine is near the $5^{\prime}$ start of exon 1. In cat, KRT71 has nine coding exons, producing a 1575-bp CDS transcript that translates into 524 amino acids. Over all exons, the cat sequence is most similar to that of dog, with an average sequence identity of $93.2 \%$ and a range of $88.6-100 \%$, and is less similar to human, with an average sequence identity of $91.7 \%$ and a range of $85.8-100 \%$. The most significant differences between the dog and cat sequences are located in exon 4. At the protein level, cat KRT71 is $95.2 \%$ identical to the dog and $93.7 \%$ identical to human. The 
Table 1 Genotyping results in cat breeds with pelage mutations

\begin{tabular}{|c|c|c|c|c|c|c|c|c|}
\hline \multirow[t]{2}{*}{ Breed } & \multirow[t]{2}{*}{$N$} & \multicolumn{3}{|l|}{ Hairless } & \multirow[t]{2}{*}{$N$} & \multicolumn{3}{|l|}{ Curly } \\
\hline & & $H R / H R$ & $H R / h r$ & $h r / h r$ & & $R E / R E$ & RE/re & relre \\
\hline American wirehair & 5 & 5 & 0 & 0 & 5 & 5 & 0 & 0 \\
\hline Devon Rex & 5 & 5 & 0 & 0 & 11 & 0 & 0 & 11 \\
\hline Devon Rex outcross & I & l & l & I & 2 & 0 & 2 & 0 \\
\hline Kohana & 3 & 1 & 1 & 1 & 3 & 2 & 1 & 0 \\
\hline Peterbald & 5 & 5 & 0 & 0 & 5 & 5 & 0 & 0 \\
\hline Domestic shorthair & 20 & 20 & 0 & 0 & 102 & 102 & 0 & 0 \\
\hline Sphynx & 34 & 0 & 8 & 26 & 34 & 28 & 6 & 0 \\
\hline Sphynx outcross & 1 & 0 & 1 & 0 & 1 & 1 & 0 & 0 \\
\hline Other curly $^{\mathrm{a}}$ & 22 & 22 & 0 & 0 & 22 & 21 & 1 & 0 \\
\hline
\end{tabular}

${ }^{a}$ Other curly includes Cornish Rex, German Rex, La Perm, Selkirk Rex, and Tennessee Rex

wild-type feline KRT71 sequence is submitted as GenBank HM564031.

KRT71 mutation analysis

The generated sequences were aligned with the publicly available sequence to identify variants. Fifty-five DNA variants, including SNPs and indels, were detected in exonic, intronic, and UTR regions in the 24 cats analyzed (Supplementary Tables 2 and 4). Fourteen SNPs were identified in the exonic regions; one variant produced a missense mutation in exon 7, c.1315G $>\mathrm{A}$, causing a serine-to-glycine substitution without a change in the charge of the amino acid. All the other silent variants were highly variable and did not appear to segregate concordantly with any hairless or rexoid phenotypes in the tested cats (Supplementary Table 2).

A complex alteration was identified in the Devon Rex cats. An 81-bp deletion (c.1108-4_1184del), including the last $4 \mathrm{bp}$ of intron 6 and the first $77 \mathrm{bp}$ of exon 7, followed by a 8-bp insertion (c.1184_1185insAGTTGGAG) and a base insertion (c.1196insT), was found to be homozygous in the two Devon Rex cats (Fig. 2).

(a)

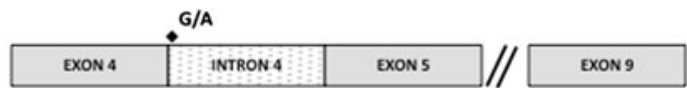

Genomic DNA

(b)

\begin{tabular}{|l|l|}
\hline EXON 4 & EXON 5 \\
\hline EXON 9 \\
\hline
\end{tabular}

Wild Type cDNA

(c)

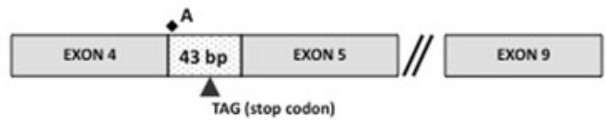

Sphynx cDNA

(d)

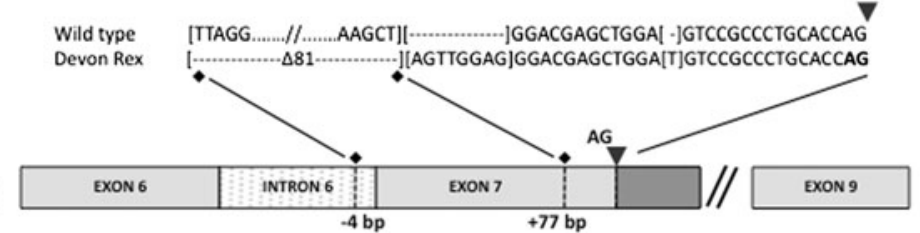

Genomic DNA

(e)

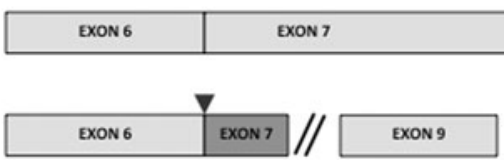

//

EXON 9

Wild Type cDNA

(f)

Fig. 2 Alternative splice models of KRT71 gene in cats. RT-PCR analysis on a control cat, a Sphynx, and a Devon Rex. a Genomic DNA with the diamond indicating the SNP [G/A] detected at position +1 of intron 4 . b Normal-haired cat cDNA with correct splicing of intron 4. c Sphynx cDNA with the 43-bp insertion caused by the disruption of the donor splice site in intron 4; triangle indicates the

\section{Devon Rex cDNA}

introduced stop codon. d Genomic DNA with diamonds indicating the region involved in the deletions/insertions causing the Devon Rex phenotype. e Normal-haired cat cDNA with correct splicing of intron 6. $f$ Devon Rex cDNA with the missing $5^{\prime}$ region of exon 7 . Triangle indicates that the exon 7 starts after the alternative splice 
Forty-one intronic SNPs were detected, and one of them at position 1 of intron 4 was identified as affecting an exon/ intron splice site in the two Sphynx cats. The c. $816+1 \mathrm{G}>\mathrm{A}$ transversion altered the $5^{\prime}$ splice site at the junction of exon 4 and intron 4 (Fig. 2). These two polymorphisms were investigated in detail.

\section{KRT71 mutation genotyping}

To determine whether the 81-bp deletion and the two insertions were responsible for the curly phenotype in Devon Rex, 185 cats were screened for this mutation by agarose gel electrophoresis. All Devon Rexes were homozygous for the identified variant; thus they are fixed for this complex mutation. One outcross Devon Rex family was tested and F1 cats with only one copy of the altered sequence had normal hair. Besides Devon Rex, the mutation was heterozygous in six Sphynx, one Kohana, and one German Rex. The KRT71 sequence alteration was absent in the randomly bred population $(N=102)$ (Table 1$)$.

The c. $816+1 \mathrm{G}>\mathrm{A}$ SNP is likely disrupting the highly conserved " $G$ " needed for the 5 ' splice site recognition signal. Within the 95 screened cats, including ten breeds with curly or hairless phenotype and randomly bred cats, the mutation was found in two breeds: Sphynx and Kohana (Table 1). One Kohana was homozygous wild type, one was heterozygous, and one was homozygous for the mutation. Within the Sphynx cats $(N=34), 26$ were homozygous for the mutation and 8 were heterozygous. Six of the eight cats were heterozygous for the Devon-associated alteration. The complete sequence was acquired from two Sphynx that carried only one copy of the "hairless" mutation and no additional alterations were identified in the obtained sequence. However, additional regions remained to be explored within the gene.

\section{KRT71 cDNA and 3' UTR analysis}

The c.1108-4_1184del involving the last 4 bp of intron 6 and the first $77 \mathrm{bp}$ of exon 7 removes the $3^{\prime}$ splice acceptor site and a new acceptor site is recognized within the same exon, r.1108_1221del. The partial amplification by RT-PCR of mRNA from the Devon skin biopsy generated a 1415-bp fragment in the wild type and a 1347-bp fragment in the Devon Rex cat, suggesting an alternative splice site acceptor within exon 7. The c. $816+1 \mathrm{G}>\mathrm{A}$ transversion in intron 4 appears to alter the $5^{\prime}$ splice site at the exon-intron junction and results in the activation of an intronic sequence: $\mathrm{r}\left[816+1 \_816+43\right.$ ins; $\left.816+1 \mathrm{~g}>\mathrm{u}\right]$. The partial amplification by RT-PCR of mRNA from skin biopsy generated a 928-bp fragment in the wild type and a 43-bp-longer transcript in the Sphynx cat, suggesting an alternative splice site donor at position 44 of intron 4 . At position 27 in the insertion, a stop codon (TGA) is generated.

\section{Discussion}

Hair is a unique structure that is characteristic of mammals and the shape of the hair follicle governs its form (Lindelof et al. 1988). The coat of the normal cat consists of three hair types: long and straight guard hairs of uniform diameter, thinner awn hairs, and fine undulating down hairs of uniform thickness. A variety of rexoid and hairless breeds are documented to have alterations in their hair types. The autosomal recessive Devon Rex coat is generally missing the guard hairs and had reduced length and thickness in the undercoat hairs. Hairless in Sphynx cats is allelic to Devon and some Sphynx have tuffs of hair on the chest, ears, or tail. Complementation testing with the other major curly breed, Cornish Rex, has shown that these autosomal recessive traits are not allelic (Robinson 1973).

Previous studies to identify the gene causing hairlessness in other species implicated KRT71 as the cause for curly coats in mice, rats, cattle, and dogs (Cadieu et al. 2009; Dunn 1937; Kikkawa et al. 2003; Poirier et al. 2002; Robinson 1981; Runkel et al. 2006; Sundberg et al. 1997; Taylor et al. 2000; A. D. Markey, unpublished). The keratin protein consists of three domains: a head domain, a low-complexity coiled-coil tail domain, and the helixforming $\alpha$-helical rod domain. The $\alpha$-helical rod domain of KRT71 plays an important role in forming heteropolymers of specific type I and type II cytokeratin through interactions of these domains (Hatzfeld and Weber 1990). In mice, mutations such as $\mathrm{Ca}^{R i n}, \mathrm{Ca}^{J}, \mathrm{Ca}^{9 J}$, and $\mathrm{Ca}^{16 J}$ induce single amino acid deletions in the $\alpha$-helical rod domain and result in curly body hair (Kikkawa et al. 2003). In rat, the $R e$ allele is identified as a 7-bp deletion at the $3^{\prime}$ splice site of intron 1 in KRT71 (Kuramoto et al. 2010). A SNP, c. $451 \mathrm{~A}>\mathrm{T}$, causing a nonsynonymous alteration, p.Arg151Trp, has been recently identified in curly hair in dogs (Cadieu et al. 2009). In cattle, an 8-bp deletion occurring in exon 1 causes an early truncated KRT71 protein resulting in a curly-hair coat (A. D. Markey, unpublished).

In the current study, KRT71 was analyzed in ten cat breeds with rexoid and hairless phenotypes. Hairless cats were included in this study because the naked phenotype of Sphynx cats is suggested to be allelic and dominant to the Devon Rex mutation (Robinson 1973). In cat, a 1575-bp nucleotide mRNA is produced and translated into 524 amino acids. Two of 55 identified mutations are likely associated with the Devon and Sphynx phenotypes. The Devon Rex mutation is a complicated set of two insertions and a deletion that leads to a splicing error. An 81-bp deletion removes the last $4 \mathrm{bp}$ of intron 6 , extending into the first 77 bp of exon 7 (c.1108-4_1184del). This deletion is followed by an 8-bp insertion of novel sequence (c.1184_1185insAGTTGGAG). This small insertion is 
followed by normal exon 7 sequence, except for one addition of thymine that is inserted $12 \mathrm{bp}$ downstream (c.1196insT). As confirmed by cDNA analysis, these mutations disrupt the $3^{\prime}$ splice site of intron 6 , the highly conserved AG, causing an alternative downstream acceptor to be employed (Fig. 2). A new alternative acceptor site is found within the first 37 bp of exon 7 (r.1108_1221del), which is $16 \mathrm{bp}$ after the single thymine insertion. The resulting protein has a 35 -amino-acid deletion at the $3^{\prime}$ $\mathrm{C}$-terminus end of the $\alpha$-helical rod domain (Supplementary Fig. 1). Approximately 200 cats were genotyped for the identified Rex (re) polymorphism and all of the Devon Rex were homozygous for the mutation. Thus, the mutation is fixed in the breed, suggesting that this mutation controls the re allele at the rex $(R)$ locus and it could be considered a breed-defining genetic characteristic. These data suggest that two copies of the deletion are required to cause a curly-coat phenotype that meets the standard for the Devon Rex breed.

A guanine-to-adenine base substitution was observed at position +1 in intron 4 in the Sphynx cats $(\mathrm{c} .816+1 \mathrm{G}>\mathrm{A})$. The guanine is a highly conserved position in the intron at the $5^{\prime}$ splice site (Hastings and Krainer 2001; Ladd and Cooper 2002). Comparison of cDNA with genomic sequences of KRT71 suggested that the GT dinucleotide sequence $44 \mathrm{bp}$ downstream of the original splice site, $\mathrm{r}\left[816+1 \_816+43\right.$ ins; $\left.816+1 \mathrm{~g}>\mathrm{u}\right]$, serves as an alternative splicing donor site in the Sphynx mutant allele (Fig. 2). Thus, an alternative splice site is recognized at base pair 44 in the modified exon 4 (Fig. 2). This alteration causes a stop codon at position 27 in the insertion, leading to a truncated KRT71 (Supplementary Fig. 1). The truncated protein deletes the majority of the $\alpha$-helical rod domain that plays an important role in forming heteropolymers of specific type I and type II cytokeratin through interactions of these domains (Hatzfeld and Weber 1990). Together, sequence alterations in exon 7 and a truncated protein affecting the $\alpha$-helical rod domain could also affect the formation of heteropolymers of specific type I and type II cytokeratins, cellular targeting, receptor binding, or proper folding of the protein after translation.

In the early stages of the hairless Sphynx breed development, crosses with Devon Rex were performed (Robinson 1973). Analysis of 34 Sphynx showed that 26 were homozygous for the intronic SNP and 8 were heterozygous for the mutation. Thus, this SNP is one of the causes of the $h r$ allele at the $H$ (hairless) locus. Six heterozygous Sphynx are compound heterozygotes for the SNP and the Devon Rex-associated deletion. These data suggest that the Sphynx allele is dominant to the Devon Rex allele, both being recessive to the wild type, supporting the phenotypic and segregation analyses previously published (Robinson 1973). However, two Sphynx were not homozygous for the $h r$ mutation nor compound heterozygous with the re mutation, suggesting that these cats have some other epistatic mutations or hairless is dominant with variable penetrance in expression.

This genetic study identified the causative mutations for two mutant alleles, $r e$ and $h r$, at two reported loci, Rex $(R)$ and Hairless $(H)$, which are actually allelic mutations in KRT71. The Sphynx mutation leads to a complete lossof-function allele where the structure of the hair is seriously compromised, i.e., the hair is produced but is easily dislodged because of the lack of a well-formed bulb. In the Devon Rex, the mutation causes a remarkable alteration in the protein as well, but evidently residual activity of the protein still exists. The KRT71 re and KRT71 ${ }^{h r}$ are the suggested allelic designations, forming the allelic series $K R T 71^{+}>K R T 71^{h r}>K R T 71^{r e}$.

Sequence analyses of KRT71 did not identify genetic alterations causative for the other six Rexoids and the other two hairless cat breeds. Since much of the intronic and regulatory regions of domestic cat KRT71 have not been analyzed, this gene has not been excluded as causative for the other phenotypic alterations. Other genes involved in the keratinization process are also possible candidates. In humans, keratin genes are clustered as families in two regions of the genome: the type I genes on chromosome 17 and the genes encoding the type II keratins on chromosome 12 (Lessin et al. 1988; Rogers et al. 1995, 2005; Rosenberg et al. 1988). Family-based or genome-wide association studies may implicate the keratin clusters for the other cat rexoid and hairless phenotypes, leaving complex studies of the specific genes to follow.

Keratins have gained increasing attention during the last couple of decades because of their causal involvement in the etiology of a large heterogeneous group of hereditary epithelial diseases (see reviews Irvine and McLean 1999; Smith 2003). Our current study demonstrates the importance of the $\alpha$-helical rod domain of KRT71 in hair formation in cats. Comparison of KRT71 mutations in various species may allow us to find associations of pelage phenotype with types of mutations and lead to better understanding of KRT71 functions. Moreover, the identification of feline coat variants should enhance the understanding of mammalian skin physiology, body homeostasis, and cell and tissue integration, and the development of a molecular test that could be used as a powerful instrument for the screening of different coat mutations will benefit the breeders.

Acknowledgments Funding for this project was provided by NIHNCRR RR016094, the Winn Feline Foundation, the Center for Companion Animal Health, School of Veterinary Medicine, University of California, Davis and the Veterinary Genetics Laboratory, University of California, Davis. We appreciate the editorial assistance from L. H. Bach and J. D. Kurushima and the numerous cat breeders 
who have provided DNA samples, especially Jeri Newman, Donna Bass, Andy Lawrence, Linda Koehl, Franklin Whittenburg, Barbara Currie, and Michelle Berg.

Open Access This article is distributed under the terms of the Creative Commons Attribution Noncommercial License which permits any noncommercial use, distribution, and reproduction in any medium, provided the original author(s) and source are credited.

\section{References}

Bighignoli B, Niini T, Grahn RA, Pedersen NC, Millon LV et al (2007) Cytidine monophospho-N-acetylneuraminic acid hydroxylase (CMAH) mutations associated with the domestic cat $\mathrm{AB}$ blood group. BMC Genet 8:27

Cadieu E, Neff MW, Quignon P, Walsh K, Chase K et al (2009) Coat variation in the domestic dog is governed by variants in three genes. Science 326:150-153

Drogemuller C, Rufenacht S, Wichert B, Leeb T (2007) Mutations within the FGF5 gene are associated with hair length in cats. Anim Genet 38:218-221

Dunn LC (1937) Caracul, a dominant mutation. J Hered 334

Hall TA (1999) BioEdit: a user-friendly biological sequence alignment editor and analysis program for Windows 95/98/NT. Nucleic Acids Symp Ser 41:95-98

Hastings ML, Krainer AR (2001) Pre-mRNA splicing in the new millennium. Curr Opin Cell Biol 13:302-309

Hatzfeld M, Weber K (1990) The coiled coil of in vitro assembled keratin filaments is a heterodimer of type I and II keratins: use of site-specific mutagenesis and recombinant protein expression. J Cell Biol 110:1199-1210

Housley DJ, Venta PJ (2006) The long and the short of it: evidence that FGF5 is a major determinant of canine 'hair'-itability. Anim Genet 37:309-315

Irvine AD, McLean WH (1999) Human keratin diseases: the increasing spectrum of disease and subtlety of the phenotypegenotype correlation. Br J Dermatol 140:815-828

Ishida Y, David VA, Eizirik E, Schaffer AA, Neelam BA et al (2006) A homozygous single-base deletion in MLPH causes the dilute coat color phenotype in the domestic cat. Genomics 88:698-705

Kehler JS, David VA, Schaffer AA, Bajema K, Eizirik E et al (2007) Four independent mutations in the feline fibroblast growth factor 5 gene determine the long-haired phenotype in domestic cats. J Hered 98:555-566

Kikkawa Y, Oyama A, Ishii R, Miura I, Amano T et al (2003) A small deletion hotspot in the type II keratin gene mK6irs1/Krt2-6g on mouse chromosome 15 , a candidate for causing the wavy hair of the caracul $(\mathrm{Ca})$ mutation. Genetics 165:721-733

Kuramoto T, Hirano R, Kuwamura M, Serikawa T (2010) Identification of the rat Rex mutation as a 7-bp deletion at splicing acceptor site of the Krt71 gene. J Vet Med Sci 72:909-912

Ladd AN, Cooper TA (2002) Finding signals that regulate alternative splicing in the postgenomic era. Genome Biol 3(11):reviews0008

Langbein L, Rogers MA, Praetzel S, Aoki N, Winter H et al (2002) A novel epithelial keratin, hK6irs1, is expressed differentially in all layers of the inner root sheath, including specialized huxley cells (Flugelzellen) of the human hair follicle. J Invest Dermatol 118:789-799

Lessin SR, Huebner K, Isobe M, Croce CM, Steinert PM (1988) Chromosomal mapping of human keratin genes-evidence of non-linkage. J Invest Dermatol 91:572-578

Lindelof B, Forslind B, Hedblad MA (1988) Human-hair formmorphology revealed by light and scanning electron microscopy and computer-aided 3-dimensional reconstruction. Arch Dermatol 124:1359-1363

Lyons LA, Foe IT, Rah HC, Grahn RA (2005a) Chocolate coated cats: TYRP1 mutations for brown color in domestic cats. Mamm Genome 16:356-366

Lyons LA, Imes DL, Rah HC, Grahn RA (2005b) Tyrosinase mutations associated with Siamese and Burmese patterns in the domestic cat (Felis catus). Anim Genet 36:119-126

Morris D (1999) Cat breeds of the World: a complete illustrated encyclopedia. Viking Penquin, New York

Poirier C, Yoshiki A, Fujiwara K, Guenet JL, Kusakabe M (2002) Hague (Hag). A new mouse hair mutation with an unstable semidominant allele. Genetics 162:831-840

Robinson R (1968) German Rex: a rexoid coat mutant in the cat. Genetica 39:351-352

Robinson R (1969) Devon Rex-a third rexoid coat mutant in cat. Genetica 40:597-599

Robinson R (1973) Canadian hairless or Sphinx cat. J Hered 64:47-49

Robinson R (1981) Rex mutant in the Norway rat. J Hered 72:131-132

Rogers MA, Winter H, Wolf C, Krieg T, Schweizer J (1995) Genomic characterization of a cluster of human type-i hair keratins, Hha2 and a novel keratin gene. J Invest Dermatol 105:469-469

Rogers MA, Edler L, Winter H, Langbein L, Beckmann I et al (2005) Characterization of new members of the human type II keratin gene family and a general evaluation of the keratin gene domain on chromosome 12q13.13. J Invest Dermatol 124:536-544

Rosenberg M, RayChaudhury A, Shows TB, Le Beau MM, Fuchs E (1988) A group of type I keratin genes on human chromosome 17: characterization and expression. Mol Cell Biol 8:722-736

Runkel F, Klaften M, Koch K, Bohnert V, Bussow H et al (2006) Morphologic and molecular characterization of two novel Krt71 (Krt2-6g) mutations: Krt71rco12 and Krt71rco13. Mamm Genome 17:1172-1182

Schmidt-Kuntzel A, Eizirik E, O'Brien SJ, Menotti-Raymond M (2005) Tyrosinase and tyrosinase related protein 1 alleles specify domestic cat coat color phenotypes of the albino and brown loci. J Hered 96:289-301

Searle AG, Jude AC (1956) The rex type of coat in the domestic cat. J Genet 506-512

Smith F (2003) The molecular genetics of keratin disorders. Am J Clin Dermatol 4:347-364

Sundberg JP, Rourk MH, Boggess D, Hogan ME, Sundberg BA et al (1997) Angora mouse mutation: altered hair cycle, follicular dystrophy, phenotypic maintenance of skin grafts, and changes in keratin expression. Vet Pathol 34:171-179

Taylor LA, Harris MJ, Juriloff DM (2000) Whiskers amiss, a new vibrissae and hair mutation near the Krt1 cluster on mouse chromosome 11. Mamm Genome 11:255-259 